\title{
Chiral symmetry and bulk-boundary correspondence in periodically driven one-dimensional systems
}

\author{
J. K. Asbóth \\ Institute for Solid State Physics and Optics, Wigner Research Centre, Hungarian Academy of Sciences, \\ P.O. Box 49, H-1525 Budapest, Hungary \\ B. Tarasinski \\ Instituut-Lorentz, Universiteit Leiden, P.O. Box 9506, 2300 RA Leiden, Netherlands \\ P. Delplace \\ Laboratoire de Physique, Ecole Normale Superieure de Lyon, 47 allee d'Italie, 69007 Lyon, France
}

(Received 12 May 2014; published 24 September 2014)

\begin{abstract}
In periodically driven lattice systems, the effective (Floquet) Hamiltonian can be engineered to be topological; then, the principle of bulk-boundary correspondence guarantees the existence of robust edge states. However, such setups can also host edge states not predicted by the Floquet Hamiltonian. The exploration of such edge states and the corresponding unique bulk topological invariants has only recently begun. In this work we calculate these invariants for chiral symmetric periodically driven one-dimensional systems. We find simple closed expressions for these invariants, as winding numbers of blocks of the unitary operator corresponding to a part of the time evolution. This gives a robust way to tune these invariants using sublattice shifts. We illustrate our ideas on the periodically driven Su-Schrieffer-Heeger model, which, as we show, can realize a discrete-time quantum walk; this opens a useful connection between periodically driven lattice systems and discrete-time quantum walks. Our work helps interpret the results of recent simulations where a large number of Floquet Majorana fermions in periodically driven superconductors have been found.
\end{abstract}

DOI: 10.1103/PhysRevB.90.125143

PACS number(s): 73.23.-b, 73.63.-b, 03.65.Vf

\section{INTRODUCTION}

Controlling the topological phases of matter is an important challenge in solid-state physics. In recent years, periodic driving has emerged as an important tool to meet this challenge. Topologically protected edge states, the hallmarks of topological phases, have been predicted and observed in periodically driven systems, such as materials irradiated by light [1-4], in shaken optical lattices [5,6], and in photonic crystals [7]. In the above cases, the principle of bulk-boundary correspondence [8] was applied to the effective (Floquet) Hamiltonian of the periodically driven system.

The variety of topological phases that periodically driven systems can display, however, is much wider than the phases of their Floquet Hamiltonians, and systematic exploration of these phases has only just begun [9]. An important example is the case of periodically driven one-dimensional topological superconductors, where the bulk $\mathbb{Z}_{2}$ invariant is replaced by a pair of $\mathbb{Z}_{2}$ invariants, whose calculation necessitates information beyond that represented by the Floquet Hamiltonian [10]. The edge states then are Floquet Majorana fermions, with potential applications in quantum information processing [11]. Such states, not predicted by the bulk Floquet Hamiltonian, have also been observed in optical realization of a one-dimensional quantum walk [12].

Simulations of one-dimensional periodically driven superconductors have shown that they can host a large number of Floquet Majorana fermions at their ends [13,14]. This can be explained by an extra chiral symmetry (CS) of the Floquet Hamiltonian, which prevents Majorana fermions on the same sublattice from recombining into complex fermions. Although this explanation is sufficient in some cases $[13,14]$, it does not describe all cases because it only relies on the Floquet Hamiltonian. Thus, the question is still open: what are the bulk topological invariants for periodically driven systems with CS?

In this paper, we find the bulk-boundary correspondence for periodically driven one-dimensional quantum systems with chiral symmetry, building on the theory of CS in discrete-time quantum walks [15-18]. We show how CS can be ensured in a periodically driven system whose time evolution in a period starts with a unitary operator $F$ by choosing an appropriate second part for the period. We show that the topological invariants predicting the number of zero- and $\pi$-quasienergy end states are the winding numbers of the blocks of $F$ in a canonical basis. Our formulas give a direct recipe to tune the topological invariants using a sublattice shift operation. We give an example of how to realize this operation in the simplest periodically driven one-dimensional Floquet insulator with $\mathrm{CS}$, the periodically driven Su-Schrieffer-Heeger (PDSSH) model. We show how this model realizes a discrete-time quantum walk and how this fact can be used to calculate the topological invariants of particle-hole symmetric quantum walks.

\section{FLOQUET FORMALISM}

We consider periodically driven single-particle lattice Hamiltonians, $H(t+1)=H(t)$. The long-time dynamics of $H(t)$, i.e., over many periods, is governed by the time-evolution operator of one period, the Floquet operator $U(\tau)$,

$$
U(\tau)=\mathbb{T} e^{-i \int_{\tau}^{\tau+1} H(t) d t},
$$


where $\mathbb{T}$ stands for time ordering. If at time $\tau$ the system is in an eigenstate $|\Psi\rangle$ of the Floquet operator, $U(\tau)|\Psi\rangle=e^{-i \varepsilon}|\Psi\rangle$, then at all times $\tau+n$, for $n \in \mathbb{Z}$, it will be in state $e^{-i n \varepsilon}|\Psi\rangle$. In this sense, the periodically driven system acts as a stroboscopic simulator of the effective (Floquet) Hamiltonian $H_{\text {eff }}$,

$$
H_{\text {eff }}(\tau)=i \ln U(\tau) .
$$

We fix the branch of the logarithm by restricting the eigenvalues $\varepsilon$ of $H_{\text {eff }}$, the quasienergies, to $-\pi<\varepsilon \leqslant \pi$.

The Floquet operator $U(\tau)$ and thus the effective Hamiltonian $H_{\text {eff }}(\tau)$ depend on the choice of the starting time of the period, $\tau$. Changing $\tau$ amounts to a unitary transformation of the Floquet operator and the effective Hamiltonian (quasienergies are independent of $\tau$ ).

\section{CHIRAL SYMMETRY OF PERIODICALLY DRIVEN SYSTEMS}

Ensuring CS of the periodically driven system amounts to ensuring that there is an initial time $\tau$ such that the corresponding effective Hamiltonian has CS; that is, there is a unitary, Hermitian, and local (within a unit cell) operator $\Gamma$ that satisfies

$$
\Gamma H_{\mathrm{eff}}(\tau) \Gamma=-H_{\mathrm{eff}}(\tau) \Leftrightarrow \Gamma U(\tau) \Gamma=U^{-1}(\tau) .
$$

The effective Hamiltonian does not inherit CS from the instantaneous Hamiltonian, which is the case for particle-hole symmetry [19]. However, CS of the periodically driven system is ensured if there is an intermediate time $0<t_{1}<1$ that splits the period into a first part and a second part in a special way. Let $F$ denote the time evolution of the first part of the cycle,

$$
F=\mathbb{T} e^{-i \int_{\tau^{\prime}}^{\tau^{\prime}+t_{1}} H(t) d t} .
$$

The second part of the cycle has to fulfill

$$
\Gamma F^{\dagger} \Gamma=\mathbb{T} e^{-i \int_{\tau^{\prime}+t_{1}}^{\tau^{\prime}+1} H(t) d t} .
$$

It is easy to check that in that case, not only $U^{\prime} \equiv U\left(\tau^{\prime}\right)$ but also $U^{\prime \prime} \equiv U\left(\tau^{\prime \prime}\right)$ have CS, where $\tau^{\prime \prime}=\tau^{\prime}+t_{1}$. These Floquet operators read

$$
U^{\prime}=\Gamma F^{\dagger} \Gamma F, \quad U^{\prime \prime}=F \Gamma F^{\dagger} \Gamma .
$$

\section{TOPOLOGICAL INVARIANTS OF THE EFFECTIVE HAMILTONIANS DUE TO CHIRAL SYMMETRY}

Consider a one-dimensional Floquet insulator: a long chain with a translation-invariant insulating bulk part whose quasienergy spectrum has gaps around $\varepsilon=0$ and $\pi$. If the system has CS, a local basis transformation can be performed that diagonalizes $\Gamma$, so that each lattice site has a sublattice index $A$ or $B$, defined via the projectors $\Pi_{A / B}=(1 \pm \Gamma) / 2$. We call such a basis a canonical basis. For the system to be a Floquet insulator, the numbers of $A$ and $B$ sites in each bulk unit cell have to be equal (or else the system would have flat bands at zero or $\pi$ quasienergy). We denote this number by $N$. In a canonical basis, the CS operator acts in each unit cell independently, as $\Gamma=\sigma_{z} \otimes 1_{N}$.

The spectrum of an effective Hamiltonian with CS is symmetric: stationary states $\left|\Psi^{\prime}\right\rangle$ of $H_{\text {eff }}^{\prime}$ with quasienergy $\varepsilon \neq 0, \pi$ have chiral symmetric partners $\Gamma\left|\Psi^{\prime}\right\rangle$ that are also eigenstates with quasienergy $-\varepsilon$. Such states can be chosen to have equal support on both sublattices. The system can also host states $\left|\Psi^{\prime}\right\rangle_{L / R}$ with quasienergy $\varepsilon=0$ or $\pi$, whose wave functions are expelled from the bulk to the left/right by the gaps in the bulk spectrum. These end states can be chosen to have support on only one sublattice.

The effective Hamiltonians $H_{\text {eff }}^{\prime}$ and $H_{\text {eff }}^{\prime \prime}$ have CS, as per Eqs. (6), and thus can be assigned topological invariants $v^{\prime}$ and $v^{\prime \prime}$. These are obtained using the standard procedure [8], whereby we first isolate the bulk part of $H_{\text {eff }}^{\prime}$ and $H_{\text {eff }}^{\prime \prime}$ by imposing periodic boundary conditions on the translationinvariant central part of these Hamiltonians and taking the thermodynamic limit. The bulk Hamiltonians are periodic functions of the quasimomentum $k \in[-\pi, \pi)$ and, in the canonical basis, are block off-diagonal,

$$
H_{\mathrm{eff}}(k)=\left(\begin{array}{cc}
0 & h(k) \\
h^{\dagger}(k) & 0
\end{array}\right) .
$$

Here and later on, $H_{\text {eff }}$ refers to either $H_{\text {eff }}^{\prime}$ or $H_{\text {eff }}^{\prime \prime}$, with similar simplifications for $U$ and $h$. The topological invariants are

$$
v^{\prime}=v\left[h^{\prime}\right], \quad v^{\prime \prime}=v\left[h^{\prime \prime}\right],
$$

where the function $v[h]$ is a winding number,

$$
\nu[h]=\frac{1}{2 \pi i} \int_{-\pi}^{\pi} d k \frac{d}{d k} \ln \operatorname{det} h(k) .
$$

These integers cannot change under adiabatic deformation of the bulk Hamiltonians, so they are equal to the winding numbers of the flat-band limits of these Hamiltonians, which are the topological invariants of Ryu et al. [8]. They can be interpreted as the dimensionless bulk sublattice polarization [20] of the effective Hamiltonians at times $\tau^{\prime}$ and $\tau^{\prime \prime}$.

\section{TOPOLOGICAL INVARIANTS OF THE DRIVEN SYSTEM}

To derive the topological invariants of the periodically driven system, we start by adopting the results obtained for discrete-time quantum walks (DTQW) with CS [18] to periodically driven systems. The derivations very closely follow those of Ref. [18], so we omit them here, but for completeness, we give details in Appendix A. As with DTQWs, in periodically driven systems, the wave functions of quasienergy $\pi$ end states also switch sublattices as they evolve from time $\tau^{\prime}$ to $\tau^{\prime \prime}$, so neither $v^{\prime}$ nor $v^{\prime \prime}$ alone gives useful information about the number of end states (observations to the contrary in specific models $[13,14]$ cannot be generalized). The winding numbers $v^{\prime}$ and $v^{\prime \prime}$ must be combined to obtain the bulk topological invariants controlling the number of end states,

$$
v_{0}=\frac{v^{\prime}+v^{\prime \prime}}{2}, \quad v_{\pi}=\frac{v^{\prime}-v^{\prime \prime}}{2} .
$$

We now proceed to simplify Eqs. (10) and express them using the blocks of $F$ in the canonical basis:

$$
F(k)=\left(\begin{array}{ll}
a(k) & b(k) \\
c(k) & d(k)
\end{array}\right) .
$$

Along the way, we will use simple properties of the function $v[A(k)]$ of Eq. (9): $v[A B]=v[A]+v[B]$ and $v\left[A^{\dagger}\right]=-v[A]$ for arbitrary $A(k)$ and $B(k)$. 
There are two constraints on the winding numbers of the blocks of the Floquet operator $F$ representing the first part of the drive cycle, both following from the unitarity of $F$. First, substituting Eqs. (11) directly into $F(k) F(k)^{\dagger}=1$ gives $a c^{\dagger}=-b d^{\dagger}$. Taking the winding numbers of the two sides gives

$$
v[c]-v[a]=v[d]-v[b] .
$$

Second, $F$ represents an operation on an open chain, terminated at its ends. Thus, the average displacement of a state in the bulk, with this average going over all possible states, has to be zero: Otherwise, unitarity of $F$ would be violated in the end regions. This average displacement is given by the winding number of $F$ itself [19], which, since $F$ is unitary, can be written as

$$
\nu[F]=\frac{1}{2 \pi i} \int d k \operatorname{Tr} F^{\dagger}(k) \frac{d}{d k} F(k) .
$$

Inserting the decomposition of $F$ in the canonical basis, Eq. (11), into $v[F]=0$ gives

$$
v[F]=v[a]+v[c]+v[b]+v[d]=0 .
$$

To use the relations derived above, we note that

$$
U=e^{-i H_{\mathrm{eff}}}=\cos H_{\mathrm{eff}}-i \sin H_{\mathrm{eff}} .
$$

Because of the block off-diagonal structure of $H_{\text {eff }}$, the first term in the sum above corresponds to the block diagonal, and the second corresponds to the block off-diagonal parts of $U$. Now since $\operatorname{sgn}(\varepsilon)=\operatorname{sgn}(\sin \varepsilon)$ for $\varepsilon \in[-\pi, \pi]$, the winding number of $H_{\mathrm{eff}}$ is the same as that of $\sin H_{\mathrm{eff}}$. Therefore, in Eq. (9), we can substitute the off-diagonal block of $U$ in a canonical basis: $h \rightarrow i U_{12}$. For the topological invariants of the effective Hamiltonians $H_{\text {eff }}^{\prime}$ and $H_{\text {eff }}^{\prime \prime}$, using Eqs. (6) and substituting the blocks of $F$, we obtain $v^{\prime}=v\left[a^{\dagger} b-c^{\dagger} d\right]$ and $v^{\prime \prime}=v\left[-a c^{\dagger}+b d^{\dagger}\right]$. We can simplify these using the unitarity of $F$, whereby $a^{\dagger} b+c^{\dagger} d=0$ and $a c^{\dagger}+b d^{\dagger}=0$, and the fact that $v[\alpha c]=v[c]$ for any $\alpha \in \mathbb{C}$. We obtain

$$
\begin{aligned}
v^{\prime} & =v[b]-v[a]=v[d]-v[c], \\
v^{\prime \prime} & =v[a]-v[c]=v[b]-v[d] .
\end{aligned}
$$

Inserting these equations into Eqs. (10), together with Eqs. (14) and (12), gives us

$$
v_{0}=v[b], \quad v_{\pi}=v[d] .
$$

These equations are the central result of our paper: In onedimensional periodically driven systems with CS, the windings of the determinant of the off-diagonal and the diagonal blocks of the Floquet operator in a canonical basis fix the number of end states at a quasienergy of 0 and $\pi$, respectively.

Equations (17) determine the topological invariant $v_{0}\left(v_{\pi}\right)$ even if the gap of $H_{\text {eff }}$ at quasienergy $\varepsilon=\pi(\varepsilon=0)$ is closed, a problem raised by Tong et al. [13]. Consider

$$
\cos H_{\mathrm{eff}}^{\prime}=1-2\left(\begin{array}{cc}
c^{\dagger} c & 0 \\
0 & b^{\dagger} b
\end{array}\right)=2\left(\begin{array}{cc}
a^{\dagger} a & 0 \\
0 & d^{\dagger} d
\end{array}\right)-1 .
$$

If there is a quasimomentum $k$ where the gap of $H_{\text {eff }}^{\prime}$ closes around $\varepsilon=0$, then $\cos H_{\text {eff }}^{\prime}(k)$ has a doubly degenerate eigenvalue of +1 . At that $k$, using the first relation of Eq. (18), either $c(k)$ or $b(k)$ (or both) has an eigenvalue of zero. This means $v_{0}$ is not well defined, and neither are $v^{\prime}$ or $v^{\prime \prime}$. However, $v_{\pi}$ from Eq. (17) is still well defined. Similarly, if at some $k$ the gap of $H_{\mathrm{eff}}^{\prime}$ around $\varepsilon=\pi$ closes, then, using the second relation of Eq. (18), $a(k)$ or $d(k)$ must have an eigenvalue of zero, and $v_{\pi}$ is not well defined, but $v_{0}$ is.

\section{GEOMETRICAL PICTURE}

In the case of a two-band one-dimensional (1D) Floquet insulator with CS, we can give a geometrical interpretation for the topological invariants $v_{0}$ and $v_{\pi}$. We relegate the details to Appendix B and just summarize the results here.

Disregarding an irrelevant global phase, the evolution operator for the first half of the period reads $F(k)=e^{-i \vec{f}(k) \vec{\sigma}}$, with $\vec{f}(k)$ being a three-dimensional real vector inside a unit sphere of radius $\pi$ whose surface points are all identified with each other and $\vec{\sigma}$ being the vector of Pauli matrices. As $k$ traverses the Brillouin zone $[-\pi, \pi[, \vec{f}(k)$ describes a directed, smooth, closed loop. If the gap around $\varepsilon=0$ is open, the loop of $\vec{f}(k)$ cannot touch the $z$ axis or the surface of the sphere, and we find that the invariant $\nu_{0}$ is given by the winding of the loop around the $z$ axis. If the gap of $H_{\text {eff }}$ around $\varepsilon=\pi$ is open, the path of $\vec{f}(k)$ cannot touch the circle in the $x y$ plane of radius $\pi / 2$. In that case, $v_{\pi}$ is given by the winding of the loop around that circle.

\section{TUNING THE INVARIANTS}

Formulas (17) allow for a simple way to tune the topological invariants of a periodically driven system, using a unitary sublattice shift operation $S(n)$, whose bulk part reads

$$
S(n, k)=\exp (-i n \Gamma k) .
$$

In the bulk, $S(n)$ displaces sites on sublattice $A(B)$ to the right (left) by $n$ sites. Therefore, at the left/right end, under the effect of $S(n), n$ states must switch sublattices, transitioning $B \rightarrow A / A \rightarrow B$ (if $n$ is negative, the opposite is true). How this transition happens depends on the details of $S(n)$, which have no influence on the topological invariants (or on the number of end states).

To tune the invariants of a periodically driven system obeying Eq. (6), with some $F=F^{(0)}$, we insert extra sublattice shifts before and after $F^{(0)}$,

$$
F^{(1)}=S(m) F^{(0)} S(n) .
$$

Substituting into Eqs. (17), we obtain directly the topological invariants of the modified driven system,

$$
v_{0}^{(1)}=v_{0}^{(0)}+m-n, \quad v_{\pi}^{(1)}=v_{\pi}^{(0)}-m-n .
$$

\section{EXAMPLE: THE PERIODICALLY DRIVEN SSH MODEL}

We now illustrate the concepts introduced above for the PDSSH model, given by

$$
H_{\mathrm{SSH}}(t)=\sum_{j=1}^{M}\left[v(t) c_{2 j} c_{2 j-1}^{\dagger}+w(t) c_{2 j+1} c_{2 j}^{\dagger}\right]+\text { H.c. },
$$

where $c_{x}$ annihilates the fermion on site $x$. For simplicity, we keep the intracell hopping amplitudes $v(t)$ and the intercell 


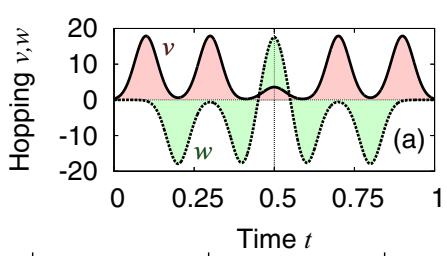

(b)
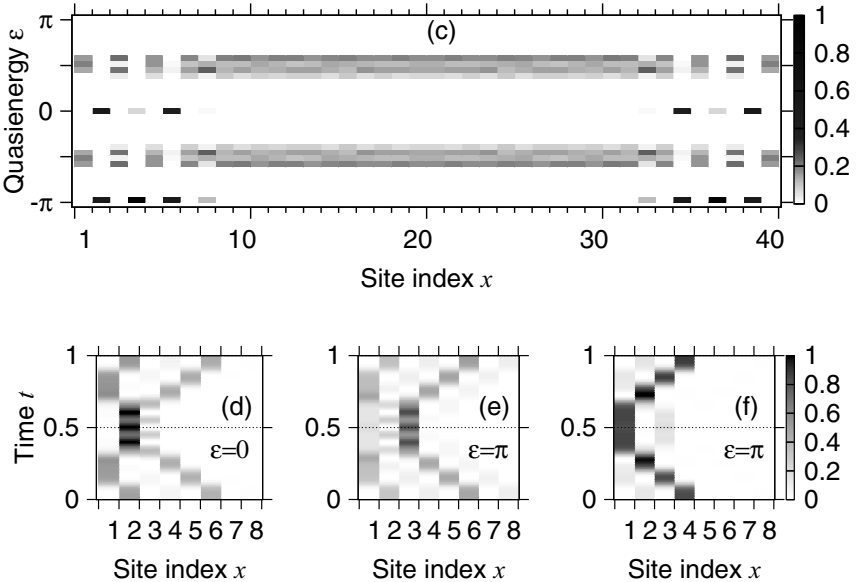

FIG. 1. (Color online) Floquet eigenstates of a periodically driven SSH chain of 40 sites. (a) Time dependence of the intracell (solid line) and intercell (dotted line) hopping amplitudes. (b) The curve $\vec{f}(k)$, which winds -1 times around the $z$ axis (red) and -2 times around the circle of radius $\pi / 2$ on the $x y$ plane, showing that $v_{0}=-1$ and $v_{\pi}=-2$. (c) Local density of states of the effective Hamiltonian $H_{\text {eff }}(0)$. (d) Time evolution of the position distribution $|\langle\Psi(t) \mid x\rangle|^{2}$ of the single end state with $\varepsilon=0$ and (e) and (f) of two orthogonal end states with $\varepsilon=\pi$.

hopping amplitudes $w(t)$ real, homogeneous in space, and modulated periodically, with period 1 . We fix open boundary conditions by identifying $c_{2 M+1}=0$ (as opposed to periodic boundary conditions, which would require $c_{2 M+1}=c_{1}$ ).

The sublattice shift operator $S(n)$ can be realized [9] by the following drive sequence: a pulse of $v$ of area $\pi / 2$, followed by a pulse of $w$ of area $-\pi / 2$. This allows us to realize a discrete-time quantum walk as a periodically driven lattice Hamiltonian.

As a concrete example, we consider the PDSSH model on an open chain of 40 sites ( $M=20$ unit cells). The drive sequence, shown in Fig. 1(a), consists of a train of nine pulses, chosen to be Gaussian for numerical convenience, applied to $v$ and $w$ homogeneously. We ensure CS by way of Eq. (6), with $t_{1}=0.5$, by choosing both $v(t)$ and $w(t)$ to be even functions of time.

We follow the recipe of Eq. (20) to realize $v_{0}=-1, v_{\pi}=$ -2 . The role of $F^{(0)}$ is played by the first half of the central Gaussian pulse, where $w=5 v$ : thus, it is a short pulse $e^{-i \pi / 2 H_{1}}$, where $H_{1}$ is an SSH Hamiltonian in the topologically nontrivial phase. So we have $v_{0}^{(0)}=1, v_{\pi}^{(0)}=0$. To test the robustness of the recipe, we realize the sublattice displacement $S(n=2)$ only approximately by allowing considerable overlaps between the $\pi / 2$ area pulses of $v$ and the $-\pi / 2$ area pulses of $w$.

We find that the bulk topological invariants and the end states agree perfectly with the theory above. The invariants are the winding numbers of the curve of Fig. 1(b), which are $v_{0}=-1, v_{\pi}=-2$. Correspondingly, in the local density of states [Fig. 1(c)], at each end, we find two end states at $\varepsilon=\pi$ and one end state at $\varepsilon=0$, exclusively localized on the $B / A$ sublattice at the left/right end. The time dependence of these end states [Figs. 1(d) and 1(f)] shows that that they indeed spread over both sublattices at intermediate times but return to a single sublattice at $t=0.5$. For the zero $(\pi)$ energy end states, this is the same (opposite) sublattice as that occupied at $t=0$.

Since we require the hopping amplitudes $v$ and $w$ to be real, the instantaneous SSH Hamiltonian, Eq. (22), has particle-hole symmetry (PHS), represented by $\Gamma K$, where $K$ denotes complex conjugation. The PDSSH model inherits this symmetry, and therefore, its end states are analogous to zero- and $\pi$-quasienergy Floquet Majorana fermions. If CS is violated but PHS is maintained, only the parity of the number of the Floquet Majorana fermions at each edge and at each quasienergy $0, \pi$ is protected. There is a corresponding pair of bulk $\mathbb{Z}_{2}$ topological invariants [10]. In the case of the PDSSH model, we can follow the construction of Jiang et al. [10], and we find that the $\mathbb{Z}_{2}$ invariants can be obtained simply from the complete areas of the pulses of $v$ and $w$. For details, see Appendix C.

\section{OUTLOOK}

The topologically protected states our theory predicts should have experimental signatures in different kinds of setups. Optical experiments, where edge states are routinely imaged directly [12,21], are in the best position to test our predictions. Alternatively, in transport measurements, the end states should give rise to transmission resonances, similar to the ones predicted for Floquet Majorana fermions [22].

Our work leaves a couple of theoretical questions open. First, is the decomposition of the drive cycle $U$ into $F$ and $\Gamma F^{\dagger} \Gamma$, as per Eqs. (4)-(6), a necessary requirement for a periodically driven Hamiltonian to have CS? For previously studied cases $[13,14]$ we can find such a decomposition, but if a counterexample were to be found, the theory we presented here would need to be expanded. Second, the bulk effective Hamiltonian $H_{\text {eff }}(\tau, k)$ of a one-dimensional Floquet insulator (with or without CS) is periodic in both $\tau$ and $k$, and thus has a Chern number. In all the examples we examined numerically, we found this Chern number to be zero, but can it take on a nonzero value? If so, what is the physical interpretation of this number? Last, how can the topological invariants we found here be formulated in the frequency domain [9]? This is especially an interesting question, as previous work on the PDSSH model using this approach [23] has not detected the pair of topological invariants we found.

\section{ACKNOWLEDGMENTS}

We thank J. Dahlhaus, J. Li, A. Gábris, and J. Edge for useful discussions. P.D. acknowledges useful comments from A. Bernevig. This research was realized in the frame of TAMOP 4.2.4. A/1-11-1-2012-0001 "National Excellence Program-Elaborating and operating an inland student and researcher personal support system," subsidized by the 
European Union and cofinanced by the European Social Fund. This work was also supported by the Hungarian National Office for Research and Technology under Contract No. ERC_HU_09 OPTOMECH and the Hungarian Academy of Sciences (Lendület Program, LP2011-016). This research was supported by the Foundation for Fundamental Research on Matter (FOM) and the Netherlands Organization for Scientific Research (NWO/OCW).

\section{APPENDIX A: DERIVATION OF EQUATIONS (10)}

To derive Eqs. (10), we closely follow the line of thought of Ref. [18]. We consider an open, periodically driven chain with $\mathrm{CS}$, which has one bulk and two ends. Let $n_{A / B, 0 / \pi}^{\prime}$ denote the number of end states at the left end on the $A / B$ sublattice at quasienergy $0 / \pi$ of the Hamiltonian $H_{\mathrm{eff}}^{\prime}$, and let $n_{A / B, 0 / \pi}^{\prime \prime}$ be the corresponding quantities for $H_{\mathrm{eff}}^{\prime \prime}$. The bulk-boundary correspondence for the effective Hamiltonians $H_{\text {eff }}^{\prime}$ and $H_{\text {eff }}^{\prime \prime}$ reads

$$
\begin{aligned}
v^{\prime} & =n_{A, 0}^{\prime}-n_{B, 0}^{\prime}+n_{A, \pi}^{\prime}-n_{B, \pi}^{\prime}, \\
v^{\prime \prime} & =n_{A, 0}^{\prime \prime}-n_{B, 0}^{\prime \prime}+n_{A, \pi}^{\prime \prime}-n_{B, \pi}^{\prime \prime} .
\end{aligned}
$$

Topologically protected end states of periodically driven one-dimensional lattices with $\mathrm{CS}$ can be divided to two classes: (i) they have quasienergy 0 and are on the same sublattice at $\tau^{\prime}$ and $\tau^{\prime \prime}$, or (ii) they have quasienergy $\pi$ and are on opposite sublattices. Indeed, consider a topologically protected end state $\left|\Psi^{\prime}\right\rangle$, which is an eigenstate of $U^{\prime}$ with eigenvalue $e^{-i \varepsilon}$, with $\varepsilon \in\{0, \pi\}$. It is only on a single sublattice: $\Gamma\left|\Psi^{\prime}\right\rangle=e^{-i \gamma}\left|\Psi^{\prime}\right\rangle$, with $\gamma=0 / \pi$ corresponding to sublattice $A / B$. Now consider the same end state at the other special time $\tau^{\prime \prime},\left|\Psi^{\prime \prime}\right\rangle=F\left|\Psi^{\prime}\right\rangle$. This is an eigenstate of $U^{\prime \prime}$ with the same quasienergy $\varepsilon$. This state is also on only one sublattice because $\Gamma F\left|\Psi^{\prime}\right\rangle=\Gamma F \Gamma e^{i \gamma}\left|\Psi^{\prime}\right\rangle=$ $\Gamma F \Gamma e^{i(\gamma-\varepsilon)} \Gamma F^{-1} \Gamma F \Psi^{\prime}=e^{i(\gamma-\varepsilon)} F\left|\Psi^{\prime}\right\rangle$. So $\left|\Psi^{\prime \prime}\right\rangle$ is on the same (opposite) sublattice as $\left|\Psi^{\prime}\right\rangle$ if $\varepsilon=0(\varepsilon=\pi)$. This can be written succinctly as

$$
\begin{gathered}
n_{A, \pi}^{\prime \prime}-n_{B, \pi}^{\prime}=n_{B, \pi}^{\prime \prime}-n_{A, \pi}^{\prime}=0, \\
n_{A, 0}^{\prime \prime}-n_{A, 0}^{\prime}=n_{B, 0}^{\prime \prime}-n_{B, 0}^{\prime}=0 .
\end{gathered}
$$

Using Eqs. (A2) to simplify $v^{\prime}+v^{\prime \prime}$ and $v^{\prime}-v^{\prime \prime}$ from Eqs. (A1), we obtain

$$
v_{0}=\frac{v^{\prime}+v^{\prime \prime}}{2}, \quad v_{\pi}=\frac{v^{\prime}-v^{\prime \prime}}{2},
$$

which are Eqs. (10), as we set out to demonstrate.

\section{APPENDIX B: GEOMETRICAL PICTURE}

For a two-band 1D Floquet insulator with CS, we can give a direct geometrical picture of the topological invariants $v_{0}$ and $v_{\pi}$. Since the global phase cannot wind ( $F$ cannot have quasienergy winding), it can safely be disregarded, and the evolution operator for the first half of the period then reads $F(k)=e^{-i \vec{f}(k) \vec{\sigma}}$. Here, $\vec{f}$ is a three-dimensional vector of magnitude $f \in[0, \pi]$, and $\vec{\sigma}$ is the vector of Pauli matrices. The $k$-dependent vector $\vec{f}(k)$ is restricted inside a spherical ball of radius $\pi$, with all points on the surface identified with each other. The parameters $a, b, c, d$ in Eq. (11) are just complex-number-valued functions of $k$,

$$
F=\left(\begin{array}{cc}
\cos f-i \sin f \cos \theta & -i \sin f \sin \theta e^{-i \phi} \\
-i \sin f \sin \theta e^{i \phi} & \cos f+i \sin f \cos \theta
\end{array}\right),
$$

using spherical coordinates. As $k$ traverses the Brillouin zone, $\vec{f}(k)$ describes a directed, smooth, closed loop that can at some $k$ exit the ball at a point on the surface and reenter at the same $k$ at the antipodal point.

If the gap around $\varepsilon=0$ is open, the loop of $\vec{f}(k)$ cannot touch the $z$ axis or the surface of the sphere. Thus, the loop has a well-defined winding number around the $z$ axis,

$$
\nu_{0}=\frac{1}{2 \pi} \int d k \frac{d}{d k} \phi(k) .
$$

Since both $f(k), \theta(k) \in] 0, \pi$ [ for all $k$, this is the same as the winding number $v_{0}$ obtained by substituting (B1) into Eq. (17).

The gap of $H_{\text {eff }}$ around $\varepsilon=\pi$ closes when $\vec{f}(k)$ is on the circle on the $n_{z}=0$ plane of radius $\pi / 2\left(n_{z}=0\right.$ and $\left.f=\pi / 2\right)$. Thus, if the gap around $\varepsilon=\pi$ is open, the loop of $\vec{f}(k)$ has a well-defined winding number around that circle. To calculate this winding number, we first discard the $\phi$ information by setting $\phi=0$. This transforms the three-dimensional closed path of $\vec{f}(k)$ into a two-dimensional path in a semicircle, with the points on the circular boundary with the same $x$ coordinate identified. We need the winding of this path around the single point $f=\pi / 2, n_{z}=0$. This is found by deforming the semicircle yet again, via the transformation $(f \sin \theta, f \cos \theta) \rightarrow(\cos f, \sin f \cos \theta)$, into a circle in whose origin the point $f=\pi / 2, n_{z}=0$ is mapped. The winding number is then

$$
\nu_{\pi}=\frac{1}{2 \pi} \int d k \frac{d}{d k} \arctan \frac{\cos f(k)}{\sin f(k) \cos \theta(k)},
$$

which is the same as $v_{\pi}$ obtained by substituting Eq. (B1) into Eq. (17b).

\section{APPENDIX C: THE $\mathbb{Z}_{2} \times \mathbb{Z}_{2}$ INVARIANT}

The PDSSH model, Eq. (22), has particle-hole symmetry, represented by $\Gamma K$, where $K$ stands for complex conjugation. This antiunitary symmetry is inherited by the effective Hamiltonian from the instantaneous Hamiltonian [19].

If we break CS in the PDSSH model, an end state can remain protected if it cannot have a PHS partner. This happens whenever the number of end states at a given energy and at a given end is odd: then, after breaking CS, a single end state is still protected by PHS. We illustrate this on the PDSSH model. If we break $\mathrm{CS}$ by delaying the intracell hopping amplitude $v$ by $\delta t$ with respect to the intercell hopping $w$ pulses, as shown in Fig. 2(a), the lone end state at $\varepsilon=0$ is still topologically protected, while the pair of end states at $\varepsilon=\pi$ hybridize and move away from the edge of the energy Brillouin zone (except for a time shift of 0.5 , where the conditions for CS are again fulfilled). To break PHS, we can add a sublattice potential to the SSH model, obtaining the periodically driven Rice-Mele (PDRM) model,

$$
H_{\mathrm{RM}}(t)=H_{\mathrm{SSH}}(t)+u(t) \sum_{x=1}^{M}\left(c_{2 x-1}^{\dagger} c_{2 x-1}-c_{2 x}^{\dagger} c_{2 x}\right) .
$$



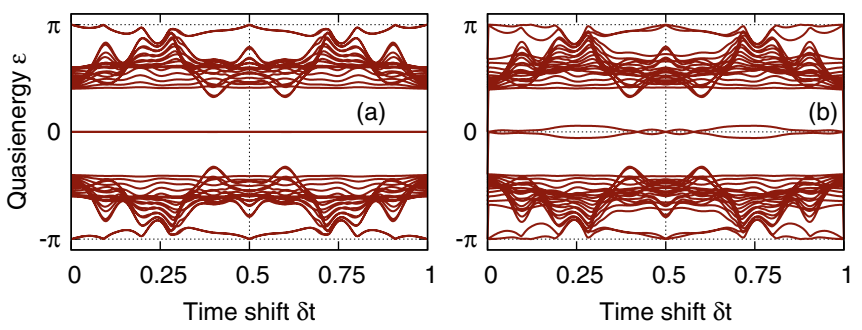

FIG. 2. (Color online) Effect of breaking CS by time shifting the pulse of the intracell hopping $v(t)$ with respect to the other pulses. (a) In the PDSSH model, the extra PHS protects the end states at $\varepsilon=0$. (b) In the PDRM model, there is no PHS, and all end-state energies are affected by the time shift.

Now, CS still holds if, in addition to $v(t)$ and $w(t)$ being even functions of time, $u(t)$ is odd: $u(t)=-u(-t)$. We choose $u(t)=\sin (2 \pi t)$. This time, if we break CS by shifting the $v(t)$ pulse in time with respect to the $w(t)$ and $u(t)$ pulses, as shown in Fig. 2(b), all end states move away from their original energies (again, except for the time shift of 0.5).

The extra PHS of the PDSSH model brings with it an extra pair of bulk topological invariants, $\left(Q_{0}, Q_{\pi}\right) \in \mathbb{Z}_{2} \times \mathbb{Z}_{2}$, which predict the number of end states protected by PHS at zero and $\pi$ energy. If we have CS, the invariants are just $Q_{\varepsilon}=v_{\varepsilon} \bmod 2$; if CS is broken, however, they can only be obtained by a procedure involving analytic continuation based on the full-cycle $H(t)$, as found by Jiang et al. [10].

We find that for the PDSSH model, the invariant of Jiang et al. [10] can be given by simple closed formulas. At the momenta $k=0$ and $k=\pi$, the Hamiltonians at different times all commute with each other, and therefore, all that matters is the total area under the $v$ and $w$ pulses,

$$
V=\int_{0}^{1} v(t) d t, \quad W=\int_{0}^{1} w(t) d t .
$$

A short calculation gives

$$
\begin{gathered}
Q_{0}=\operatorname{sgn}\left(\sin \frac{V+W}{2} \sin \frac{V-W}{2}\right), \\
Q_{0} Q_{\pi}=\operatorname{sgn}[\sin (V+W) \sin (V-W)] .
\end{gathered}
$$

\section{APPENDIX D: MAPPING TO THE DISCRETE-TIME QUANTUM WALK}

The PDSSH model, besides being the simplest periodically driven topological insulator, also gives a lattice realization of the discrete-time split-step quantum walk. For the quantum walk, we need to define the basis states $|R / L, x\rangle$ for a coin state predicting the next step to the right/left and the walker at position $x$. These basis states are identified with states on the $\mathrm{SSH}$ chain as

$$
\begin{gathered}
c_{2 x+1}^{\dagger}|0\rangle=|R, x\rangle, \\
c_{2 x}^{\dagger}|0\rangle=-i|L, x\rangle .
\end{gathered}
$$

The basic operations of the split-step walk are rotations of the internal state of the walker, $R(\theta)=e^{-i \theta \sigma_{y}}$, and shifts of the $R / L$ internal state to the right/left, given by $S_{ \pm}=e^{-i k\left(\sigma_{z} \pm 1\right)}$. One time step of the split-step walk is defined as

$$
U=S_{-} e^{-i \theta_{2} \sigma_{y}} S_{+} e^{-i \theta_{1} \sigma_{y}} .
$$

A pulse of $v$ of area $V$ followed by a pulse of $w$ of area $W$ in the basis of Eq. (D2) can be written as

$$
U=e^{-i W\left(\cos k \sigma_{y}-\sin k \sigma_{x}\right)} e^{-i V \sigma_{y}},
$$

which reproduces the time step of the split-step walk with the angles

$$
\theta_{2}=W+\pi / 2, \quad \theta_{1}=V-\pi / 2 .
$$

The above mapping is important as it allows us to apply results for the topological phases of periodically driven systems to quantum walks.

As an example, consider the invariants due to $\mathrm{CS}$, via Eqs. (17), for the simple quantum walk, given by $U=$ $S_{-} S_{+} e^{-i \theta \sigma_{y}}$. According to the mapping above, the winding numbers are $v_{0}=v\left[-i\left(s+c e^{i k}\right], v_{\pi}=v\left[c-s e^{-i k}\right]\right.$, with $c=\cos (\pi / 4+\theta / 2), s=\sin (\pi / 4+\theta / 2)$. We get $\left(v_{0}, v_{\pi}\right)=$ $(+1,0)$ if $|c|>|s|$, i.e., if $\theta \in[-\pi, 0]$, and $(0,-1)$ if $\theta \in[0, \pi]$. This is shifted by $(1 / 2,-1 / 2)$ from the invariants obtained by the scattering matrix method [24], but such a shift is not physical: both methods predict a pair of end states at zero and $\pi$ quasienergy at an interface between bulks with $\theta<0$ and $\theta>0$, as seen in simulations [17].

Another example is the calculation of the invariants due to PHS in the split-step quantum walk. Compared to the invariants $Q_{0}^{\text {(gap) }}, Q_{\pi}^{\text {(gap) }}$, defined via gap closings in the parameter space [17], the above mapping to the PDSSH model, together with Eqs. (C4), gives $Q_{0}=Q_{0}^{\text {(gap) }}$ and $Q_{\pi}=1-Q_{\pi}^{\text {(gap) }}$, which agrees in all the predictions concerning end states at interfaces. Comparing the results to the scattering matrix topological invariants [24], we, of course, find the same constant shift by $(1 / 2,-1 / 2)$ as that for the invariants due to $\mathrm{CS}$, which has no influence on the physical predictions.
[1] N. H. Lindner, G. Refael, and V. Galitski, Nat. Phys. 7, 490 (2011).

[2] B. Dóra, J. Cayssol, F. Simon, and R. Moessner, Phys. Rev. Lett. 108, 056602 (2012).

[3] T. Kitagawa, T. Oka, A. Brataas, L. Fu, and E. Demler, Phys. Rev. B 84, 235108 (2011).

[4] Y. H. Wang, H. Steinberg, P. Jarillo-Herrero, and N. Gedik, Science 342, 453 (2013).
[5] P. Hauke, O. Tieleman, A. Celi, C. Ölschläger, J. Simonet, J. Struck, M. Weinberg, P. Windpassinger, K. Sengstock, M. Lewenstein et al., Phys. Rev. Lett. 109, 145301 (2012).

[6] M. D. Reichl and E. J. Mueller, Phys. Rev. A 89, 063628 (2014).

[7] M. C. Rechtsman, J. M. Zeuner, Y. Plotnik, Y. Lumer, D. Podolsky, F. Dreisow, S. Nolte, M. Segev, and A. Szameit, Nature (London) 496, 196 (2013). 
[8] S. Ryu, A. P. Schnyder, A. Furusaki, and A. W. W. Ludwig, New J. Phys. 12, 065010 (2010).

[9] M. S. Rudner, N. H. Lindner, E. Berg, and M. Levin, Phys. Rev. X 3, 031005 (2013).

[10] L. Jiang, T. Kitagawa, J. Alicea, A. R. Akhmerov, D. Pekker, G. Refael, J. I. Cirac, E. Demler, M. D. Lukin, and P. Zoller, Phys. Rev. Lett. 106, 220402 (2011).

[11] D. E. Liu, A. Levchenko, and H. U. Baranger, Phys. Rev. Lett. 111, 047002 (2013)

[12] T. Kitagawa, M. A. Broome, A. Fedrizzi, M. S. Rudner, E. Berg, I. Kassal, A. Aspuru-Guzik, E. Demler, and A. G. White, Nat. Commun. 3, 882 (2012).

[13] Q.-J. Tong, J.-H. An, J. Gong, H.-G. Luo, and C. H. Oh, Phys. Rev. B 87, 201109 (2013).

[14] M. Thakurathi, A. A. Patel, D. Sen, and A. Dutta, Phys. Rev. B 88, 155133 (2013).
[15] T. Kitagawa, M. S. Rudner, E. Berg, and E. Demler, Phys. Rev. A 82, 033429 (2010).

[16] T. Kitagawa, Quantum Inf. Process. 11, 1107 (2012).

[17] J. K. Asbóth, Phys. Rev. B 86, 195414 (2012).

[18] J. K. Asbóth and H. Obuse, Phys. Rev. B 88, 121406 (2013).

[19] T. Kitagawa, E. Berg, M. Rudner, and E. Demler, Phys. Rev. B 82, 235114 (2010).

[20] I. Mondragon-Shem, T. L. Hughes, J. Song, and E. Prodan, Phys. Rev. Lett. 113, 046802 (2014).

[21] Y. E. Kraus, Y. Lahini, Z. Ringel, M. Verbin, and O. Zilberberg, Phys. Rev. Lett. 109, 106402 (2012).

[22] A. Kundu and B. Seradjeh, Phys. Rev. Lett. 111, 136402 (2013).

[23] A. Gómez-León and G. Platero, Phys. Rev. Lett. 110, 200403 (2013).

[24] B. Tarasinski, J. K. Asbóth, and J. P. Dahlhaus, Phys. Rev. A 89, 042327 (2014). 